\title{
THE RAPID ISOLATION OF MYCOBACTERIUM TUBERCULOSIS AND THE SIMULTANEOUS STREPTOMYCIN SENSITIVITY DETERMINATION
}

\author{
BY \\ A. F. MACCABE AND J. C. GOULD \\ From the Department of Bacteriology, University of Edinburgh
}

(RECEIVED FOR PUBLICATION JULY 30, 1953)

One of the difficulties in the rapid laboratory diagnosis of tuberculosis is the time required for a satisfactory growth of the tubercle bacillus to appear on artificial medium. This delay is even more important when it is also desirable to determine the sensitivity of the organism. While in some cases it may not be of paramount importance to obtain a rapid growth of the tubercle bacillus for routine diagnosis, the result of the sensitivity test to be of any great value to the clinician must be available in the shortest possible time. Therefore, the ideal to be aimed at should be the rapid isolation of the organism and the simultaneous determination of its sensitivity.

The medium used for isolating $M$. tuberculosis in many laboratories is that of LowensteinJensen. This medium is satisfactory, but growth of the organism is not usually obtained in less than 14 to 28 days. Slide culture in saponated blood (Giammalvo, Natsios, and Elton, 1949) is more rapid, but the method is too laborious and requires considerable skill. Tarshis and Frisch (1951a and b) and Frisch and Tarshis (1951) demonstrated the value of human blood in culturing the tubercle bacillus.

For sensitivity determinations the serial dilution tube method (M.R.C. Report, 1948) has been widely used but again it is time consuming, and it is necessary to have a culture of the organism already growing. The slide culture method, although accurate, also takes too much time, and is potentially dangerous in a busy laboratory. The incorporation of antibiotic in solid medium is used but requires specially prepared medium and duplicates, both for control purposes and in testing a range of concentrations. The diffusion method of Tinne and Henderson (1950) is convenient but it is essential to keep the bottle upright during the whole period of the test. A more convenient reservoir of the antibiotic is the compressed tablet (Hoyt, 1951), the filter paper strip (Fairbrother and Southall, 1951), or the filter paper disc (Gould and Bowie, 1952).

This paper describes a method whereby the isolation and sensitivity of the tubercle bacillus can be determined simultaneously by adopting the filter paper disc technique and a saponated human blood agar medium. This medium was suggested by Dr. W. M. Levinthal, of the Bacteriology Department, Astley Ainslie Institute, Edinburgh, and, after development by one of us, was found to give more rapid results than the Lowenstein-Jensen medium.

\section{Media}

Saponated Blood Agar.-To $95 \mathrm{ml}$. of citrated group $\mathrm{O}$ human blood, $5 \mathrm{ml}$. of $10 \%$ saponin is added, and the mixture heated to $55^{\circ}$ for five minutes. Equal quantities of saponated blood and a 4\% agar solution (not nutrient) are then mixed at $45^{\circ}$. If kept in the refrigerator this medium will remain suitable for 10 days.

Lowenstein-Jensen Solid Medium (Mackie and McCartney, 1948).-This medium was sloped in $10 \mathrm{ml}$. amounts in 1-oz. "universal" containers, the containers being held horizontal during solidification. Petri dishes containing 5-mm. thicknesses were also used.

Dubos Fluid Medium (Dubos and Davis, 1946).This was kept in 1-oz. containers.

\section{Inocula}

Cultures. $-M$. tuberculosis H37Rv and other strains were maintained on Lowenstein-Jensen medium and saponated blood agar. The growth was emulsified in the condensation water as evenly as possible, and one standard loopful (gauge wire 2-mm. diameter loop) of this suspension was used as inoculum for each test by spreading evenly over the surface of the medium. Similarly, one standard loopful of Dubos culture was used as an inoculum.

Sputa.-These were all from patients suffering from tuberculosis, and, before use, were examined by Ziehl- 
TABLE I

DIFFERENCE IN RATE OF GROWTH OF TUBERCLE BACILLUS ON SAPONATED BLOOD AGAR MEDIUM COMPARED WITH LOWENSTEIN-JENSEN MEDIUM

\begin{tabular}{|c|c|c|c|c|c|c|c|c|c|c|c|c|c|c|c|}
\hline \multirow{2}{*}{ Inoculum } & \multirow{2}{*}{$\begin{array}{c}\text { No. of } \\
\text { Specimens } \\
\text { Examined }\end{array}$} & \multirow{2}{*}{$\begin{array}{l}\text { No. of } \\
\text { L.J. More } \\
\text { Rapid than } \\
\text { S.B.A. }\end{array}$} & \multirow{2}{*}{$\begin{array}{c}\text { No. of } \\
\text { Growth } \\
\text { Appearing } \\
\text { at Same Time }\end{array}$} & \multicolumn{12}{|c|}{ No. where S.B.A. More Rapid than L.J. (Days) } \\
\hline & & & & 1 & 2 & 3 & 4 & 5 & 6 & 7 & 8 & 9 & 10 & $10+$ & Total \\
\hline $\begin{array}{l}\text { Sputa } \\
\text { Cultures }\end{array}$ & $\begin{array}{r}115 \\
50\end{array}$ & $\begin{array}{l}0 \\
0\end{array}$ & $\begin{array}{l}25 \\
15\end{array}$ & $\begin{array}{l}1 \\
2\end{array}$ & $\begin{array}{l}4 \\
5\end{array}$ & $\begin{array}{l}4 \\
2\end{array}$ & $\begin{array}{r}11 \\
6\end{array}$ & $\begin{array}{l}14 \\
10\end{array}$ & $\begin{array}{l}5 \\
1\end{array}$ & $\begin{array}{l}5 \\
3\end{array}$ & $\underline{2}$ & 6 & $\begin{array}{l}6 \\
4\end{array}$ & $\begin{array}{r}32 \\
2\end{array}$ & $\begin{array}{l}90 \\
35\end{array}$ \\
\hline
\end{tabular}

Neelsen film. Only those specimens which were positive on film examination were accepted. No attempt was made to grade the sputa.,++++++ , etc. The accepted sputa were concentrated by the acid method. The inoculum was one standard loopful of the concentrate.

\section{Dises}

These were prepared from Whatman's No. 1 filter paper (Gould and Bowie, 1952; Bowie and Gould, 1952).

A series of discs of varying strengths was prepared ranging from 0.5 to $100 \mu \mathrm{g}$. streptomycin per disc.

Each disc was applied to the centre of the culture area immediately after inoculation. The bottle caps were replaced and Petri dishes sealed with "sellotape." All cultures were incubated at $37^{\circ}$ and examined daily for growth and zones of inhibition.

\section{Rate of Growth of $M$. tuberculosis on Saponated Blood Agar and Lowenstein-Jensen Media}

Table I shows that in 90 out of 115 consecutive specimens of sputa the tubercle bacillus grew more rapidly on saponated blood agar than on Lowenstein-Jensen medium. The difference varied from one to more than 10 days. In eight tests the difference was more than 20 days. In five tests no growth was obtained on Lowenstein-Jensen medium, whereas all saponated blood agar cultures produced growth.

The interesting point is that in no test did Lowenstein-Jensen medium produce more rapid growth than saponated blood agar. In 12 of the 25 tests where no difference in the time of appearance of growth was observed, the saponated blood agar medium produced a +++ growth compared with a + on Lowenstein-Jensen.

Fig. 1 illustrates the differences in the time of appearance of growth on the two media.

Table I also shows that out of 50 pure cultures of the tubercle bacillus obtained from sputa in no case did growth appear earlier on Lowenstein-Jensen medium. In 15 instances there was no difference in time between growth on the two media, but even in those the growth was much $\vec{\omega}$

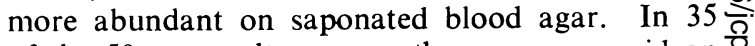
of the 50 pure cultures growth was more rapid on saponated blood agar, the difference varying from one to more than 10 days. Fig. 2 illustrates the $\dot{c}$ difference in the time of appearance of growth on $\mathrm{O}$ the two media.

\section{Streptomycin Sensitivity Tests on Saponated Blood Agar and Lowenstein-Jensen Media}

In these tests the thickness of the medium in the Petri dishes and bottles was approximately $\vec{\oplus}$ uniform.

The zones of inhibition of growth were determined with the $\mathrm{H} 37 \mathrm{Rv}$ strain as test organism, and varying strengths of streptomycin per disc. The results are noted in Table II.

\section{Preparation of the Standard Graph}

From the results obtained in Table II a graph혹 was drawn by plotting the diameter of the zones $\frac{3}{\sqrt{3}}$ of inhibition against the logarithm of the strepto-政 mycin concentration per disc. The graph is shown in Fig. 3.

\section{Deduction of Sensitivity from the Standard} Graph

With a disc containing an antibiotic there will be a concentration gradient from the disc out-을

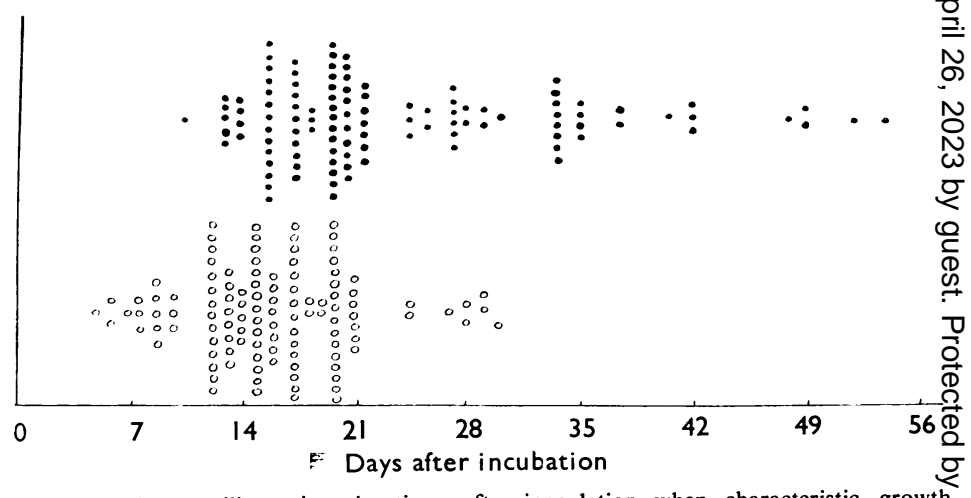

FIG. 1.-Diagram illustrating the times after inoculation when characteristic growt of $M$. tuberculosis first appeared in 115 specimens of sputum. Jensen medium. Saponated blood agar medium. 
TABLE II

ZONES OF INHIBITION OF GROWTH OF M. TUBERCULOSIS WITH STREPTOMYCIN DISCS OF VARYING STRENGTH

\begin{tabular}{c|c|c}
\hline $\begin{array}{c}\text { Streptomycin } \\
(\mu \mathrm{g} . \text { per Disc) }\end{array}$ & $\begin{array}{c}\text { No. of } \\
\text { Tests }\end{array}$ & $\begin{array}{c}\text { Average Diameter } \\
\text { Zone of Inhibition } \\
\text { on S.B.A. and L.J. } \\
\text { (mm.) }\end{array}$ \\
\hline $0 \cdot 5$ & 10 & No zone \\
1 & 20 & 7 \\
2 & 21 & 10 \\
4 & 23 & 13 \\
6 & 13 & 17 \\
8 & 15 & 19 \\
20 & 105 & 21 \\
30 & 22 & 29 \\
50 & 16 & 31 \\
100 & 16 & 42 \\
\hline
\end{tabular}

wards, and the zone of inhibition obtained with any test organism will depend on the sensitivity of that organism.

A zone of inhibition of the same diameter as that obtained with the standard organism signifies that the concentration required to inhibit the test organism is the same as that required for the standard organism. If the zone of inhibition with the test organism differs from that obtained with the standard organism the test organism requires a greater or lesser concentration for inhibition than the standard organism.

The sensitivity, i.e., the minimal inhibitory concentration, of any unknown test organism is calculated by reference to the amount of antibiotic per disc required to inhibit the standard organism to the same zone diameter. The sensitivity of our

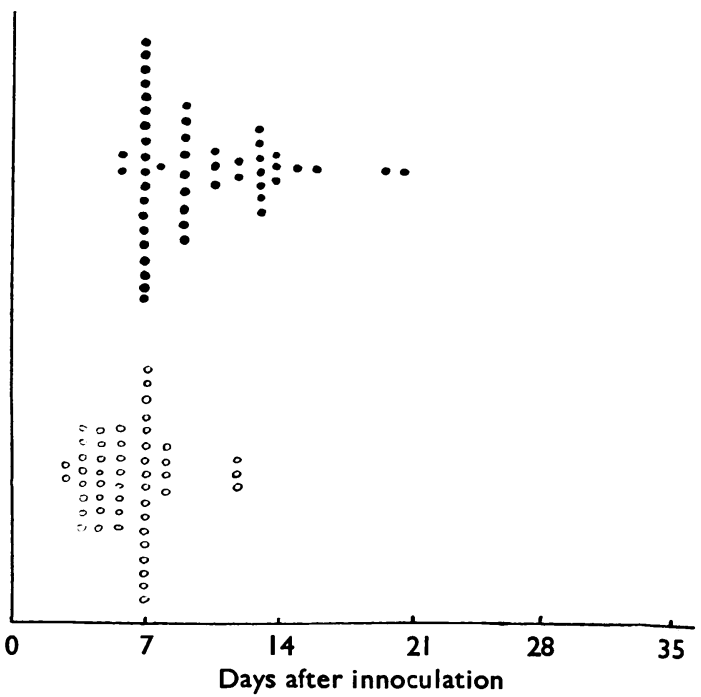

FIG. 2.-Diagram illustrating the times after inoculation when characteristic growth first appeared in subcultures of 50 strains of $M$. tuberculosis. Lowenstein-Jensen medium. O Saponated blood agar medium.

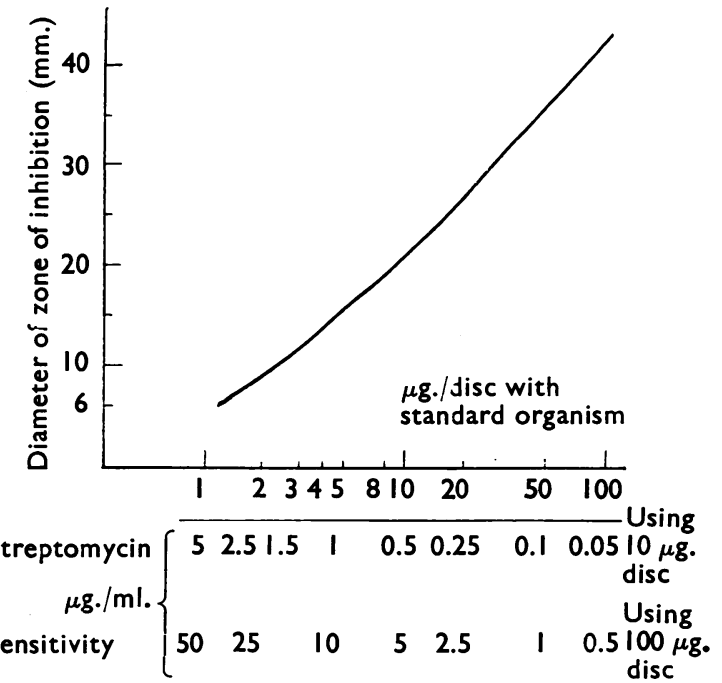

FIG. 3.-Standard graph for streptomycin.

standard M. tuberculosis $\mathrm{H} 37 \mathrm{Rv}$ is $0.5 \mu \mathrm{g}$. per $\mathrm{ml}$. of streptomycin. The sensitivity of the test organism is therefore equal to:

$$
\begin{gathered}
\text { Amount per disc required to inhibit test organism } \\
\hline \text { Amount per disc required to inhibit standard } \\
\text { organism to the same distance }
\end{gathered} \times \begin{aligned}
& \text { Sensitivity } \\
& \text { standard } \\
& \text { organism }
\end{aligned}
$$

For example: suppose the diameter of the zone of inhibition of a test organism with a standard $10 \mu \mathrm{g}$. streptomycin disc is $15 \mathrm{~mm}$., then the graph indicates that a disc containing $4 \mu \mathrm{g}$. streptomycin will inhibit the standard organism to $15 \mathrm{~mm}$. Therefore the sensitivity of the test organism is equal to $\frac{10}{4} \times 0.5=1.25 \mu \mathrm{g}$. per $\mathrm{ml}$.

Effect of Variable Inoculum.-Sensitivity tests were carried out with the standard strain inoculum varied some tenfold. The results showed that the zones of inhibition of growth did not vary more than $2 \mathrm{~mm}$.

The disc containing $10 \mu \mathrm{g}$. of streptomycin, as used for other streptomycin sensitivity tests in this laboratory, was adopted for routine use.

\section{The Standard Test}

This may be carried out in one of two ways.

(1) Directly from the sputum: a standard loopful of a specimen of concentrated sputum is inoculated on saponated blood agar or Lowenstein-Jensen slopes as described, and a $10 \mu \mathrm{g}$. streptomycin disc applied to the centre of the inoculated area.

The bottles are closed and incubated in the upright position at $37^{\circ}$ and examined daily. The zones of inhibition of growth are noted, and reference made to the standard graph. 
(2) Pure cultures are used as inoculum and the procedure is as in (1). Sensitive and resistant control strains are also set up with each series of tests.

Time of Reading the Test.-It was noted with several strains of tubercle bacilli tested that the zone of inhibition of growth on saponated blood agar medium tended to become smaller and smaller with continued incubation, whereas on LowensteinJensen medium a zone of inhibition remained.

\section{Discussion}

The work reported in this paper demonstrates the ddvantages of human saponated blood agar as a medium for the isolation of $M$. tuberculosis from material showing the presence of acid-fast bacilli. This medium is easy to prepare and may be stored for an appreciable time in the refrigerator.

The tables show that saponated blood agar medium can yield a quicker growth of the tubercle bacillus than Lowenstein-Jensen medium. It is significant that in no case did Lowenstein-Jensen medium yield a more rapid growth than saponated blood agar; in five tests Lowenstein-Jensen medium failed to show any growth at all, and the saponated blood agar showed growth in every case.

In 36 tests with microscopically positive sputa a growth was seen on saponated blood agar in under 14 days, the shortest time being four days in one case. On Lowenstein-Jensen medium there were only six tests where growth was observed in under 14 days, the shortest time being 11 days in one case. For these reasons it is concluded that saponated human blood agar is a better medium than Lowenstein-Jensen medium for culturing the tubercle bacillus.

The reasons for the saponated blood medium supporting growth of the tubercle bacillus better than Lowenstein-Jensen medium are not known. It may be that there are additional growth factors for the organism present in the blood that are not present in the egg; the saponin, as a surface-active agent, may promote more rapid cell division and so earlier visible growth. Further studies are being carried out on this problem.

Disadvantages of the medium are that the colour of the growth is not so strikingly contrasted with the colour of the medium, but a little experience soon enables one to detect growth in the earliest stages; the medium is not so inhibitory to other organisms likely to be found in sputa and exudates under test so that some care is required in the preparation of inocula direct from pathological material. However, very few cases of contamination were encountered in this series of tests.
The characteristics of the colony of the tubercle bacillus on saponated blood agar are of interest ${ }_{0}^{-}$ Early growth is shown by small, circular, convex colonies with an entire edge, bluish-grey and fairlye. moist. After about three weeks the centre of them colony changes to brown and eventually this change spreads to the periphery. In about six weeks the colony starts to become dry and $\overrightarrow{0}$ irregular, but never to such a degree as seen on $\overrightarrow{ }$ Lowenstein-Jensen medium. Some colonies after two months exhibit a depressed centre. At this stage, the colony is about $2 \mathrm{~mm}$. in diameter.

Hoyt (1951) and Gould and Bowie (1952) havealready shown that the disc diffusion test is adapt $\frac{-1}{\mathrm{c}}$ able to antibiotic sensitivity determination foro M. tuberculosis. The slow growth of the organism is no drawback to this type of test since we believe that diffusion outward from the disc is very rapiob and, if a bactericidal concentration is reacheo within a certain radius, it will kill all the cells inoculated on the medium within this area. Thus a zone of inhibition of growth results which is independent of the time of incubation and subse:quent diffusion of the antibiotic.

With sputa containing a sufficiently high densitys of bacilli the standard test with direct inoculum is possible, giving a result at or about the same time as the result of diagnostic culture. This increases the usefulness of sensitivity tests in the treatmen $\overrightarrow{\vec{b}}$ of tuberculosis with antibiotic substances. The effect of the quantitative variation in inoculum on the results of such tests is probably of some impor tance, though not so great as might be expected Even so, the results are likely to have some value as an indication for therapy, because they may be confirmed by subculture sensitivity tests, whicis remain the more accurate estimation of in vitræ sensitivity.

Most of the tests carried out and reported in this series were in the nature of subculture testso With both Lowenstein-Jensen and saponated blood agar media the striking feature is the repros ducibility of the results under reasonably standard conditions. These conditions may be maintained in the routine laboratory, and the reproducibility lends confidence to the interpretation of the results. Thus, with standard discs in use it has been found possible to make the test quantitatives using reference graphs which are detailed in the text, and bringing the sensitivity determination foo $M$. tuberculosis into line with the test devised fo other bacteria (Gould and Bowie, 1952).

From the graphs it will be seen that with a $10-\mu$ g streptomycin disc a range of sensitivity from 0 to $5 \mu \mathrm{g} . / \mathrm{ml}$. is obtained. This range will include $\mathrm{a} B$ 
those strains of $M$. tuberculosis which are usually described as sensitive to streptomycin, and with a sensitivity value within the therapeutic concentration range usually attained. For these reasons we have adopted the disc of $10 \mu \mathrm{g}$. streptomycin for our standard test, and the graph is marked accordingly. Discs containing a greater amount of the antibiotic, e.g., $100 \mu \mathrm{g}$., may be used to cover a wider range of concentrations.

This standard test may be carried out in any routine laboratory without an appreciable increase in labour, and with either Lowenstein-Jensen, saponated blood agar, or similar medium. Only one bottle of the medium is required for the test, together with a bottle for control growth without a disc if desired. Both sensitive and resistant control tests are also set up with each series of tests. No special skill is necessary in setting up the test, and only a little extra time to that required for routine diagnostic culture. Moreover, the results are easy to read.

A most interesting observation is that the zones of inhibition of growth on saponated blood agar are sometimes smaller than with the same strain on Lowenstein-Jensen medium, and this may manifest itself only after the tenth day, when there is an apparent growing in of the organisms towards the disc on the saponated blood agar. This finding may be in agreement with those of Fisher (1952) and Mitchison (1953), who found that strains of $M$. tuberculosis resistant to isonicotinic acid hydrazide tend to lose the capacity for growing on simpler media, and require extra factors for growth. In such instances, resistance to a drug may only be shown on media that contain these factors; these may be present in saponated blood and not in Lowenstein-Jensen medium, and this may be correlated with our evidence showing saponated blood agar to be a much more conducive medium for the tubercle bacillus, lessening the generation time and so enabling the organism to adapt itself more rapidly to grow in the presence of streptomycin, for example, by the adoption of an alternative metabolism.

Alternatively, the difference in the zone of inhibition may illustrate streptomycin as a bacteriostatic, rather than a bactericidal, agent ; this would occur more readily on saponated blood agar, where the growth of the organism is more favoured than on Lowenstein-Jensen medium.

Thus strains apparently resistant to streptomycin on saponated blood agar are probably resistant in vivo to the concentration covered by the test, though apparently fully sensitive on LowensteinJensen or Dubos medium. It must be remem- bered that in such an in vitro test the results must be interpreted with caution, and are not necessarily a true reflexion of the interaction of the bacillus, host, and antibiotic. Up to the present, the correlation of in vitro sensitivity of the tubercle bacillus with the clinical result of therapy has not been of a very high standard (Gernez-Rieux, Sévin, and Chénet, 1949). It is possible that the results of tests on saponated blood agar may be more closely correlated with the clinical results of treatment, and this is under investigation.

\section{Summary}

A saponated blood agar medium is described for the rapid isolation of $M$. tuberculosis.

The tubercle bacillus was isolated from 115 consecutive specimens of sputa microscopically positive more rapidly on this medium than with Lowenstein-Jensen medium, the difference being more than 20 days in some cases. Fifty strains of $M$. tuberculosis subcultured on saponated blood agar grew more rapidly than when subcultured on Lowenstein-Jensen medium. In no instance did Lowenstein-Jensen medium give more rapid growth than saponated blood agar.

The streptomycin sensitivity of the tubercle bacillus was determined on saponated blood agar and on Lowenstein-Jensen media, the disc diffusion technique being used. This test may be carried out on cultures direct from the specimen, and simultaneously read along with the result of primary culture. Alternatively, the test can be carried out with pure cultures of tubercle bacilli.

The diameters of the zones of inhibition have been related to the inhibitory concentrations of streptomycin, and the sensitivity of each organism may be found by using the graph shown.

The significance of the results is discussed.

We express our thanks to Prof. T. J. Mackie for his continued interest and advice, and to Dr. A. T. Wallace for specimens of sputa.

\section{REFERENCES}

Bowie, J. H., and Gould, J. C. (1952). Journal of Clinical Pathology, $5,356$.

Dubos, R. J., and Davis, B. D. (1946). J. exp. Med., 83, 409.

Fairbrother, R. W., and Southall, J. E. (1951). Journal of Clinical Pathology, 4, 183.

Fisher, M. W. (1952). Amer. Rev. Tuberc., 66, 626.

Fisher, M. W. (1952). Amer. Rev. Tuberc., 66, 626.
Frisch, A. W., and Tarshis, M. S. (1951). Ibid., 64, 551.

Gernez-Rieux, C., Sévin, A., and Chénet, C. (1949). Ann. Inst. Pasteur Lille, 2, 57. Giammalvo, J. T., Natsios, G. A., and Elton, N. W. (1949). Amer.

Gould, J. C., and Bowie, J. H. (1952). Edinb. med. J., 59, 178.

Moyt, R. E. (1951). Antibiot. and Chemother., 1, 469. Bacteriology, 8th ed., p. 172. Livingstone, Edinburgh.

Mitchison. D. A. (1953). Journal of Clinical Pathology, 6, 118. M.R.C. Report (1948). Lancet, 2, 862.

Tarshis, M. S., and Frisch, A. W. (1951a). Amer. J. clin. Path., 21, 101 .

Tinne, J. E., and Henderson, J. L. (1950). Lancet, 2, 901. 